Results (1) Most of the people have a moderate maximal potential platelet activity except $7.1 \%$ with very low level and $14.3 \%$ with very high level potential platelet activity. (2) Through residual platelet activity detecting, it can be seen that patients who are sensitive in ADP pathway are not necessarily sensitive in $\mathrm{AA}$ pathway, and vice-versa. (3) The frequency percent from clopidogrel is $22.0 \%$ in inhibition rate of platelet activation less than $50 \%$ group, $18.8 \%$ in that more than $90 \%$ group. But that from aspirin is $14.6 \%$ and $26.5 \%, p=0.0778$ between the two pathways.

Conclusions Potential platelet activities are not same in different patients; Residual platelet activities are also different; Even for the same patient, the platelet inhibition ability derived from Aspirin is different from that from Clopidegrel. These individual differences in platelet activity further prove the necessity for case by case analysis and remedy adjustment.

\section{e0325 INFLUENCE OF PROBUCOL ON ENDOTHELIAL DEPENDENT VASODILATION REACTION IN PATIENTS WITH ACUTE CORONARY SYNDROME}

doi:10.1136/hrt.2010.208967.325

Hong-Mei Dong, Lan Huang, Yao-Ming Song, Ai-Min Li, Jun Jin, Gang Zhao, ChunMei Lin, Chun-rong Tao. Xinqiao Hospital, Third Military Medical University

Objective The study tested the antioxidant probucol for endothelial dependent vasodilation reaction in the patients of ACS after received therapy.

Methods 51 ACS patients were divided randomly into a probucol treatment group ( $p$ ) and a routine treatment group (C). Oxidised low-density lipoprotein (ox-LDL) was measured in peripheral blood by Sandwich ELISE method. The brachial arterial hyperaemiainduced flow mediated dilation (FMD) and sublingual nitroglycerin (NTG) mediated vasodilation were measured by high resolution ultrasound. These variables were analysed after received probucol 3 months.

Results The level of FMD with probucol treatment group was significantly increased after 3 months compared to pretreamented $(p<0.05)$, while the level of ox-LDL in peripheral blood in ACS patients was markedly diseased $(p<0.01)$. The FMD with probucol treatment group was significantly increased compared to ACS routine treatment group $(p<0.05)$, while the level of ox-LDL was markedly desaesed $(p<0.01)$. The linear correlation analysis showed that plasma ox-LDL was a negative correlation with FMD in after treatmented patients by probucol with ACS $(r=-0.517, p<0.001)$.

Conclusions It was important that vascular endothelial function and endothelial dependent vasodilation reaction may be improved in the ACS patients of after the antioxidant probucol therapy.

\section{e0326 EFFECTS OF HYDROGEN SULFIDE ON PROLIFERATION OF BONE MARROW DERIVED ENDOTHELIAL PROGENITOR CELLS IN MICE}

doi:10.1136/hrt.2010.208967.326

Li Wei, Li Ai-min, Wang Hang, Zhu Jin-kun, Huang Lan. Department of Cardiology, Xinqiao Hospital, Third Military Medical University, Chongqing, China

Hydrogen sulfide $\left(\mathrm{H}_{2} \mathrm{~S}\right)$ is found to be the third endogenous gaseous transmitter and plays an important role in many systems of organism. In cardiovascular system, $\mathrm{H}_{2} \mathrm{~S}$ is produced endogenously by cystathionine gamma-lyase (CSE). The $\mathrm{CSE} / \mathrm{H}_{2} \mathrm{~S}$ system executes the physiological function of vasorelaxation, inhibition of vascular remodelling and cardioprotection. It is also concerned with variety of cardiovascular diseases such as hypertension and pulmonary hypertension. These results suggest that $\mathrm{H} 2 \mathrm{~S}$ may be a novel cardiovascular functional regulator. And, at another hand, EPCs incorporate into the process of injured carotid reendothelialisation. EPCs transplantation induces an increase in the circulating EPCs, accelerates the process of endothelial repairmen and reduces neointima formation.

Objective To study whether hydrogen sulfide has effects on endothelial progenitor cells (EPCs)

Methods Total mononuclear cells (MNCs) isolated from bone marrow by density gradient centrifugation combined with adherence cells filtration were plated on fibronect in coated culture dishes. After 7 days, adherent cells were kept with different concentrations of hydrogen sulfide for $48 \mathrm{~h}$. EPCs proliferation, migration ability and adhesion assay was performed: EPCs apoptosis was induced by paclitaxel or serum starvation for $48 \mathrm{~h}$, apoptosis was determined by TUNEL method and flow cytometry.

Results Incubation of hydrogen sulfide dose dependently increased the number of EPCs $(p<0101)$ : hydrogen sulfide improved EPCs proliferation, migration and adhesive capacity $(p<0.01)$, and hydrogen sulfide could protect EPCs from paclitaxel or serum starvation-induced apoptosis $(\mathrm{p}<0.01)$.

Conclusions Hydrogen sulfide can increase the number of bone marrow derived EPCs and improve their biological characteristics.

\section{e0327 PREVENTION THROMBOEMBOLISM USING WARFARIN FOR}

doi:10.1136/hrt.2010.208967.327

Li Xiaoming, Zhang Yuean, Gao Bingbing, Li Bao, Wu Weihua. Shanxi Cardiovascular Disease Hospital

Objective To identify the risk factors of thromboembolism related to atrial fibrillation (AF) in difference structural heart disease and prevention of thromboemboembolism with warfarin.

Methods 285 consecutive cases with AF, of which 110 were rheumatic heart disease (RHD), and 57 received prosthetic heat valve, and 53 received medicine therapy; of which 67 were hypertension; of which 35 were patients without structural heat disease; of which 26 were patients with sick sinus syndrome who are receiving a pacemaker for symptomatic bradycardia of which 16 were ischaemic heart disease; of which 15 were dilated cardiomyopathy; of which 10 were congenital heart disease; of which 8 were other disease. Clinical data including gender; age; incidence of left atrial (LA) thrombus; incidene of thromboembolism or history of stroke or transient ischaemic attack (TIA); incidence of using warfarin and dose of warfarin; events of death.

Results Incidence of left atrial (LA) thrombus (21.8\%) and incidence of thromboembolism or history of stroke or transient ischaemic attacck (TIA) $(9.1 \%)$ in RHD group were significantly higher than those in other group $(0-4 \%)(p<0.001)$; incidence of using warfarin in RHD group ( $94.5 \%)$ were significantly higher than those in other group $(61.7 \%)(\mathrm{p}<0.05)$.

Conclusion Antithrombotic therapy is key and essential treatment in AF with one high or two moderate risk factors. RHD and prosthetic heart valve is high risk factor of LA thrombus and thromboembolism. 7 patients of AF with hypertension and ischaemic heart disease have thromboembolism, but only one patient by transthoracic echocardiography (TTE) have LA thrombus. So transesophageal echocardiogram is done. AF in whom anticoagulation is indicated to use warfarin. The target International Normalised Ratio (INR) range is 2.0-3.0, but while combination of warfarin and amiodarone the dose of warfarin is less than average dose. 